\title{
Les prêts entre proches ou l'invisibilité des transactions intimes
}

Gilles Lazuech

\section{(2) OpenEdition}

\section{Journals}

Édition électronique

URL : http://journals.openedition.org/ress/1093

DOI : $10.4000 /$ ress. 1093

ISSN : $1663-4446$

Éditeur

Librairie Droz

Édition imprimée

Date de publication : 15 juin 2012

Pagination : 41-62

ISBN : 978-2-600-01613-1

ISSN : 0048-8046

\section{Référence électronique}

Gilles Lazuech, « Les prêts entre proches ou l'invisibilité des transactions intimes », Revue européenne des sciences sociales [En ligne], 50-1 | 2012, mis en ligne le 15 juin 2015, consulté le 19 avril 2019.

URL : http://journals.openedition.org/ress/1093 ; DOI : 10.4000/ress.1093 


\title{
LES PRÊTS ENTRE PROCHES OU L'INVISIBILITÉ DES TRANSACTIONS INTIMES
}

GILLES LAZUECH

Université de Nantes - Centre Nantais de Sociologie gilles.lazuech@univ-nantes.fr

\begin{abstract}
Résumé. Cet article se propose de s'intéresser aux relations de prêts entre personnes de connaissance. Si la relation d'octroi de prêts dans le cadre des banques et des institutions de crédits semble maintenant avoir été assez largement investiguée, tant par les économistes que par les sociologues, ceci ne semble pas être encore le cas des relations de prêts entre proches (famille et amis). Au cours du développement, notre texte visera à objectiver cette catégorie de prêts en partant de l'analyse des «scènes sociales» dans lesquelles ils sont réalisés. Puis, en conclusion nous chercherons à donner un sens plus général à des relations d'argent qui ne sont ni marchandes ni totalement gratuites.
\end{abstract}

Mots-clés: crédits entre proche, relation de confiance, économie morale, scène sociale.

\begin{abstract}
This article intends to focus on the relationships that are built when there is a loan between people who are acquainted with each other. If relationships when a loan is granted in a bank or credit institution have been largely investigated by both economists and sociologists, it has not been done yet in cases of loans between relatives or friends. Our forthcoming article aims at objectivizing the latter, basing itself primarily on the analysis of the "social scenes" during which these loans are granted. Then, to come to a conclusion, we shall attempt to give a broader meaning to moneycentered relationships which have neither a market value nor are they entirely free.
\end{abstract}

Keywords: loans between relatives or friends, trustful relationship, moral economy, social scene. 
INTRODUCTION

À l'occasion d'une enquête statistique portant sur les usages quotidiens de l'argent, 4I \% des personnes interrogées avaient déclaré prêter régulièrement de l'argent à un proche, $32 \%$ l'avoir déjà fait mais de façon moins systématique et $27 \%$ ne l'avoir jamais fait'. Ces prêts très courants, banals dans certains cas, enchâssés dans les relations de proximités, sont généralement de «petits prêts » qui s'effectuent entre personnes de connaissances et dont le degré de codification et de formalisation semble assez bas.

Le tableau présenté ci-dessous permet d’affiner notre approche en prenant en compte quelques indicateurs sociaux classiques pour l'analyse sociologique ${ }^{2}$. On peut constater, tout d'abord, que les prêts entre proches, ou prêts d'interconnaissance, ne concernent pas une catégorie particulière d’agents sociaux statistiquement repérée par son âge, son niveau de revenu, ses diplômes ou encore par son genre. Ainsi, lesjeunes déclarent prêter de l'argent aussi fréquemment que les plus âgés, les catégories populaires aussi souvent que les cadres ou les membres des professions libérales, les ménages disposant de revenus modestes autant que ceux qui sont plus aisés.

Des différences apparaissent cependant entre les prêteurs en observant plus attentivement les pourcentages présentés dans le tableau. Ainsi les femmes prêtent plus souvent que les hommes ( $43 \%$ contre $38 \%$ ). Cette différence s'explique sûrement par le rôle encore central qu'elles occupent dans la gestion au quotidien de l'argent domestique. Selon nos observations, pour des sommes inférieures à 50 euros, elles sont plus souvent sollicitées que les hommes, du moins par leurs enfants. On doit préciser, par ailleurs, que si les prêts sont aussi fréquents aux deux extrémités de l'échelle sociale, les sommes engagées ne sont pas les mêmes ainsi que la raison des prêts. En nous appuyant sur d’autres résultats statistiques ainsi que sur des témoignages collectés au cours de plusieurs enquêtes portant sur les usages quotidiens de l'argent (Lazuech, 20I2), les ménages disposant d'un revenu élevé (supérieur pour un couple à 5000 euros par mois) sont surreprésentés pour la catégorie des prêts supérieurs à I oo० euros, alors que ce sont les petits prêts qui

I Voir ci-dessous le dispositif général de l'enquête.

2 Pourcentages calculés hors les non-réponses qui, pour cette question, étaient inférieures à $3 \%$. 
l'emportent pour les ménages aux revenus plus modestes. Si ces derniers prêtent de moins grosses sommes, ils déclarent cependant prêter plus souvent à une personne n’appartenant pas directement à leur famille que ne le font les catégories plus aisées. Pour ces dernières, semble-t-il, les prêts se font principalement entre les membres d'un même groupe domestique. On observe également des différences entre les catégories sociales et/ou de revenu à l'occasion d'un événement précis comme celui que constitue, par exemple, le départ des enfants du domicile parental. Au sein des catégories populaires les prêts de «dépannage » consentis aux enfants l'emportent sur les prêts «d’installation» (achat de meubles, appareils électroménagers, etc.) qui caractérisent plutôt les classes moyennes supérieures³.

Prêter ou donner de l'argent à un proche

\begin{tabular}{|c|c|c|c|}
\hline & FRÉQUEMMENT & EXCEPTIONNELLEMENT & JAMAIS \\
\hline HOMMES & $38(24)$ & $34(29)$ & $28(53)$ \\
\hline FEMMES & $43(26)$ & $30(22)$ & $27(52)$ \\
\hline JEUNES DE MOINS DE 25 ANS & $48(22)$ & $27(35)$ & $25(43)$ \\
\hline ADULTES ENTRE 35-55 ANS & $35(24)$ & $34(19)$ & $31(57)$ \\
\hline ADULTES DE PLUS DE 65 ANS & $45(32)$ & $29(23)$ & $26(45)$ \\
\hline REVENU DU COUPLE INFÉRIEUR À 2000€ & $36(18)$ & $28(19)$ & $36(62)$ \\
\hline REVENU DU COUPLE COMPRIS ENTRE 3000 ET 5000€ & $34(23)$ & $37(23)$ & $29(54)$ \\
\hline REVENU DU COUPLE SUPÉRIEUR À 5000€ & $42(26)$ & $43(26)$ & $15(45)$ \\
\hline $\begin{array}{c}\text { CATÉGORIES SOCIALES APPARTENANT A LA CATÉGORIE } \\
\text { DES CADRES SUPÉRIEURS ET DES PROFESSIONS LIBÉRALES }\end{array}$ & $43(32)$ & $36(24)$ & $21(44)$ \\
\hline CATÉGORIES SOCIALES POPULAIRES & $43(23)$ & $29(20)$ & $28(57)$ \\
\hline
\end{tabular}

(hors argent de poche et argent de «soutient» versé régulièrement ${ }^{4}$ ) - Lire: $38 \%$ des hommes interrogés ont déclaré prêter fréquemment de l'argent à un proche et $24 \%$ en donner fréquemment.

3 Cette différence s'explique, en partie, par les modes de résidence choisis. Pour les jeunes salariés des catégories sociales supérieures le choix résidentiel se porte davantage vers l'occupation d'un logement individuel, avec ou sans co-location. Les jeunes qui sont d'origine sociale populaire se retrouvent plus fréquemment dans des logements collectifs et/ou sociaux: Foyer de Jeunes Travailleurs, Résidences Jeunes ou encore le Foyer-Soleil.

4 La plupart des adolescents de 13 à 17 ans, selon une enquête réalisée par nos soins, reçoivent en 2010 de l'argent de poche. Par argent de «soutien» nous entendons les versements réguliers que font des parents (ou des grands-parents) à leurs enfants majeurs alors même qu'ils occupent un emploi salarié. 
Au delà des différences entre les groupes sociaux, ce qui caractérise les prêts entre proches, plus sûrement que les dons d'argent (pourcentages entre parenthèses dans le tableau), c'est leur fréquence. Ces petits prêts, qui ne donnent que rarement lieu à des traces écrites (reconnaissance de dette), ne sont pas spontanément évoqués par les personnes rencontrées lors des entretiens alors que les prêts bancaires le sont presque systématiquement. Pour la plupart, ceux qui ont contractés un crédit auprès d'une banque et/ou d'une institution de crédit savent assez précisément le montant de la somme empruntée, le nombre d'anuitées de remboursement ainsi que les sommes mensuelles à rembourser. Pour les prêts entre proches, particulièrement pour les «petites» sommes, tout se passe comme s'ils étaient devenus invisibles, comme s'ils avaient pour caractéristique de se diluer au sein des choses du quotidien, celles qui seraient classées sans importance. Des réponses lapidaires comme, «entre amis on ne compte pas», «si on ne s'aide pas qui le fera», «c'est normal, c'est la vie», «faut bien se rendre des services», etc. confortent cette première analyse.

Les prêts d’argent, au même titre que les services rendus à titre gratuit au sein des familles ou des proches, sont donc constitutifs de la normalité des relations familiales et amicales (Fougeyrollas-Schwebe, 1994). Selon les travaux consacrés par le Crédoc aux solidarités familiales, 89\% des Français auraient été aidés par leur famille au cours de l'année 2007, dont 4I \% sous la forme d'une aide financière. $87 \%$ des personnes interrogées par le Crédoc se disent prêtes à aider financièrement l'un de leurs proches. Les jeunes (I 8-30 ans) reçoivent plus souvent des aides financières que les autres groupes d'âge, en particulier celui des adultes de plus de 65 ans qui, à l'inverse, est un groupe particulièrement aidant (Bigot, 2007). Ces aides, qui peuvent être des prêts ou des dons, contribuent aussi à faire vivre réellement les relations entre proches: les histoires de familles (et d'amitiés) sont également des histoires dans lesquelles l'argent intervient à un moment ou à un autre. Nous reviendrons sur cette dimension parce qu'elle est importante pour comprendre la nature sociale de certaines relations d'argent qui s'effectuent en famille, où entre personnes de connaissance, mais qui ne s'observent pas ailleurs ou alors de façon beaucoup plus affaiblie (à l'occasion des prêts bancaires notamment). 
Dans ce texte nous essaierons de mieux objectiver certaines des spécificités des prêts entre proches. Spécificités qui les constitueraient comme des prêts «autrement » en comparaison avec celles qui s'observent à l'occasion de formes plus institutionnalisées d'octroi de crédit. Nous essaierons aussi de mieux caractériser une catégorie d'échanges qui ne relève ni de la relation marchande, ni du don pur.

\section{DES TRANSACTIONS INVISIBLES?}

Peu de travaux empiriques ont été consacrés en sciences sociales au thème des prêts entre proches à l'exception de quelques recherches en ethnographie économique et en histoire économique (Fontaine, 2008; Weber, 2007). L'«argent des familles », pour désigner par ces termes un ensemble assez vaste de travaux, a été principalement abordé en six grands thèmes qui peuvent se recouper (Blanc, 2008). Sans procéder à un recension exhaustive de ces travaux, on peut distinguer ceux qui mettent en avant les aspects genrés des usages de l'argent, ceux qui portent sur les solidarités intrafamiliales, des travaux qui s'intéressent à l'autonomie financière des jeunes adultes, d’autres qui mettent en évidence que les transferts d’argent entre générations accentuent les inégalités sociales, un ensemble de travaux qui porte sur la reproduction économique et symbolique des familles à l'aide de la mobilisation du capital économique et, enfin, ceux souvent produits par des économistes qui interrogent la redistribution intergénérationnelle du revenu familial.

Par leur modestie (les prêts les plus fréquents sont inférieurs à 50 euros) les prêts entre proches n'ont pas fait l'objet d'un travail spécifique autour des six grands thèmes évoqués (l'argent de poche relevant, pour sa part, plus de la catégorie des dons que de celle des prêts). La discrétion des travaux empiriques en sciences sociales fait d’ailleurs écho à celle des études réalisées que ce soit par les instituts de la statistique publique ou par les banques ou les institutions de crédit. Le chercheur intéressé par les prêts d’interconnaissance ne peut donc s'appuyer que très partiellement sur des données produites par l'INSEE ou le $\mathrm{CREDOC}^{5}$. Il n’a pas non plus à sa disposition l'équivalent des études réali-

5 Le CREDOC a réalisé pour 2006 et 2007 un baromètre des solidarités familiales qui aborde de manière un peu générale les transferts d'argent intrafamiliaux dans le cadre de la solidarité. 
sées par l'Observatoire de l'endettement des ménages ou de l'Observatoire des crédits aux ménages (OCM) par exemple. Mais, comme nous le soulignons en introduction, l'invisibilité des transactions intimes est produite, voire renforcée, par les agents concernés. Considérées souvent sans importance, à l'exception des «gros » prêts, l'enquêteur rencontre beaucoup d'obstacles lorsqu'il se donne pour tâche d'objectiver ce type de relations d'argent.

Ces différentes remarques conduisent à penser que les données statistiques que nous avons produites et qui servent à appuyer notre raisonnement sont très contestables. En effet, outre leur mode de construction, leur fiabilité dépend de la bonne volonté des répondants à renseigner des questions que certains considèrent comme intrusives et/ou n'ayant pas beaucoup de sens pour eux. Le tableau qui a été présenté en introduction, et qui est construit à partir de divers indicateurs contenus dans une enquête réalisée auprès de I Ioo personnes dans les départements de Loire-Atlantique et de Vendée, renseigne certes sur des pratiques mais aussi sur la perception et la compréhension qu'ont eu les personnes répondantes aux questions posées dans le questionnaire et ceci sans explication préalable de la part des enquêteurs. Lors des entretiens de face à face, les écarts de perception et de compréhension entre l'enquêté et l'enquêteur sont apparus à de nombreuses reprises. Si spontanément la plupart des personnes que nous avons rencontrées considèrent, par exemple, que prêter 500 euros à un membre de sa famille constitue effectivement un prêt qui vient aider un proche, ces mêmes personnes sont moins nombreuses à penser qu'avancer zo euros à un bon collègue de travail relève de la catégorie des prêts. Le plus souvent, si l'on concentre l'observation sur les pratiques, un prêt est considéré comme tel par le prêteur - selon ses propres catégories de perception - lorsqu'il est, d'une façon ou d'une autre, «enregistré » (soit mentalement, soit de façon écrite) et lorsque l'obligation de rendre est explicite. Or, pour la catégorie des «petits prêts» beaucoup de prêteurs nous ont confié ne pas établir de «traces» et ne pas toujours signifier, au moment de la transaction, l'obligation de rendre. 


\section{Le dispositif général d'enquête}

Cet article s'appuie principalement sur les résultats d'une enquête par questionnaire qui a été réalisée au cours des années 2005 à 2007 selon la méthode des quotas. I 100 personnes ont été interrogées, 169 questions ont été posées. Cette enquête visait à recueillir des informations sur les pratiques de l'argent et sur les représentations de l'argent, sur les relations avec la banque et les conseillers financiers, sur la place de l'argent au sein du couple ou de la famille. Les données quantitatives ont permit de réaliser une seconde enquête au cours des années 2008 à 2010 auprès d'une trentaine de ménages. Cette seconde enquête, dite qualitative, a été inspirée par la «méthode par cas» (Passeron, Revel, 2005). Les familles rencontrées l'ont été moins eu égard à des critères de représentativité statistique qu'en fonction de l'intérêt qu'elles présentaient pour l'enquête. Si certaines familles ont été contactées parce que nous disposions de leurs coordonnées (demandées à l'issue du questionnaire d'enquête statistique), d'autres sont des personnes de connaissances: amis, membres de la famille, voisins, etc. choisies en fonction de l'intérêt de leur situation et de leurs pratiques d'argent.

La nature «invisible» des prêts entre proches, du moins de la majorité d'entre eux, nous a conduit au fil des recherches à mobiliser la notion de parenté pratique élaborée par Florence Weber ${ }^{6}$ (2005) afin de pouvoir en rendre compte. En nous appuyant sur la notion de parenté pratique il nous a été possible de penser que si certaines des relations d'argent sont peu visibles, c'est parce qu'elles sont enchâssées au cœur de la normalité du quotidien. L'invisibilité de ces prêts serait donc constitutive de relations d'échanges considérées comme «normales » (Durkheim, 2005) au sein des groupes d'interconnaissance caractérisés par un degré élevé d’intimité sociale.

6 Par parenté pratique est désigné l'ensemble des pratiques qui se déroulent à l'occasion des échanges les plus ordinaires de la quotidienneté des familles. Les relations familiales, mais on peut penser aussi aux relations amicales, se construisent à travers de multiples échanges qui, pour la plupart, ne relèvent pas de l'ordre économique. 


\section{CRÉDITS D'ÉTABLISSEMENT, CRÉDITS D'INTERCONNAISSANCE}

Pour mettre à jour l'une des différences ontologiques qui distingue les prêts entre proches de ceux accordés par les différents établissements de crédit on peut mobiliser la notion polanyienne d'encastrement (Polanyi, 1994). Les prêts entre proches sont encastrés dans des relations d'interconnaissances qui sont aussi, le plus souvent, des relations affectives. Ils relèvent d'un mode de justification qui appartient à la «cité domestique» (Boltanski, Thévenot, 199I), alors que les crédits d'établissement sont, pour leur part, encastrés dans l'ordre marchand. Au sein de cet ordre, l'argent acquiert le statut de marchandise et les relations marchandes sont instrumentales. Les prêts d’argent consentis aux particuliers n'ont d'autre justification, pour l'institution qui les octroie, que celle de la logique du profit - ou de la rentabilité - ce qui n’est pas le cas des prêts entre proches pour lesquels l'argent n'est pas une marchandise puisque la relation, quoique monétaire, n’est pas marchande (Testart, 200I). L'exemple le plus frappant où la «froide» relation impersonnelle, marquée uniquement par la logique de l'intérêt marchand, s'applique presque parfaitement est celui des crédits revolving. Opérations de crédit à l'occasion desquelles certains établissements pratiquent des taux d'intérêts parfois supérieurs à $20 \%$, taux que l'on pourrait qualifier d'usuraire. Certes la ferme distinction entre les crédits d'établissement et les crédits d'interconnaissance souffre de notables exceptions. Des établissements de crédit, via les aides de l'État ou des différentes collectivités territoriales, peuvent consentir des prêts aux particuliers avec des taux d'intérêts très bas, voire nuls (prêts bonifiés, prêts à taux zéro, etc.). Depuis quelques années des formes nouvelles d'octroi de crédits ont pu se développer, comme les crédits de particulier à particulier, les prêts «solidaires » ou encore ceux relevant de la micro-finance. Les emprunteurs qui font appel au microcrédit social bénéficient d’un accompagnement personnalisé qui, dans certains cas, peut s'apparenter à une relation de proximité alors que fondamentalement la relation reste de nature marchande (Glémain, 20Io). Dans le cadre des relations d'interconnaissance, on peut aussi donner, ici ou là, quelques exemples ou le prêteur profite de sa position pour imposer à l'emprunteur 
des garanties et des rémunérations qui peuvent parfois paraître totalement injustifiées (Bourdieu, 1996). La différence entre ce qui relève de l'économie domestique et des autres formes d'économie est parfois ténue contrairement à ce qu’affirment ceux qui restent attachés à la distinction entre les «sphères séparées » et qui pensent les différents espaces sociaux en «mondes hostiles » (Zelizer, 2005). Comme le souligne Pascal Chantelat (2002), il y a aussi des relations personnelles, voire affectives au sein des relations marchandes et, parfois, des motivations très marchandes au sein des relations personnelles.

Les mots utilisés, même de façon courante, renvoient ou désignent des «réalités» qui pour certaines semblent objectives - «un arbre» est un arbre -, alors que d'autres termes semblent renvoyer à des réalités plus subjectives, comme les mots «amour» «amitié» ou «confiance». Le terme crédit est à l'intersection de ces deux «réalités » : accorder un crédit est une réalité objective, puisque évaluable en argent, mais qui se construit généralement sur une réalité subjective, celle de la confiance, pensée comme une qualité associée (ou non) à un individu. Comme le précise Laurence Fontaine (2008), accorder son crédit, c'est croire en l'autre. C’est sur ce point précis, qui lie le crédit à la confiance, que nous voudrions observer les particularités des prêts d'interconnaissance par rapport aux prêts d'établissement (Guianne, 20ıo). Au sujet des contrats de crédit, l'économiste Dorothée Rivaud-Danset montre que lorsqu'un client sollicite un prêt, la banque n'est pas certaine qu'il pourra la rembourser. Elle se trouve en situation d'aléa moral, la non connaissance de ce que sera le futur de son client introduit une incertitude, donc un risque (Rivaud-Danset, 1996). Pour s'en prémunir, le banquier dispose d'outils - le scoring - qui lui permettent de recueillir des renseignements sur la situation passée et présente de son client (âge, état de santé, situation matrimoniale, situation professionnelle, régularité des revenus, fréquence des découverts, etc.). Ce dispositif abstrait de production de la confiance a été favorisé par le processus de «modernisation» des banques qui se manifeste, depuis les années 1980, par une plus grande segmentation de la clientèle, une technicisation accrue de la relation aux clients et une volonté des directions générales d'unifier les procédures d'octroi de crédits pour l'ensemble des conseillers clientèle d'une même 
enseigne. La relation de proximité entre le client et son conseiller qui pouvait encore s'observer dans les années 1960 se distend, même si elle demeure ici ou là (Courpasson, 1995). Moins bien connu de son conseiller, le client doit donc faire la preuve qu'on peut lui faire confiance, la relation bancaire se construit alors sur des dispositifs abstraits dont le scoring est un des éléments constitutifs? ${ }^{7}$. La construction de la confiance, comme lien social, est aussi au cœur des prêts d'interconnaissance. La confiance est rarement aveugle, même envers un proche ${ }^{8}$, et si la plupart des prêteurs disent avoir a priori confiance en l'emprunteur, tous ceux avec qui nous avons abordé ce sujet, en particulier pour des prêts importants, ont mis en avant les risques éventuels qu’ils encouraient : «Il faudrait que ce soit une personne de confiance, soit mes enfants, soit des amis. La personne à qui j'avancerai de l'argent, je dois être sûr qu'elle me remboursera» (Chauffeur routier, 48 ans) ; «De toute façon, il faudrait que le motif soit essentiel, si c'est pour s'offrir des vacances alors c'est non» (Professeur des écoles, 53 ans) ; «Je prêterais sans hésiter si c'est vraiment un ami qui est en très grande difficulté, ça sera pas une question d’argent mais une question d’amitié... Après, ça sera pas pris à la légère, ça peut être qu’une décision de couple» (Avocate, 40 ans) ; «Peut-être que je prêterai, mais je trouve ça malsain d'avoir des dettes avec des amis, surtout si elles durent dans le temps. » (Cadre technique, 37 ans). On observe, en lisant ces différents verbatim, que ce

7 À propos de la relation bancaire, on pourra se reporter aux différentes contributions suivantes: Michel Ferrary, 1999, «Confiance et Accumulation de capital social dans la régulation des activités de crédit», Revue française de sociologie, XL-3, p. 559-586; Jeanne Lazarus, 20II, L'Épreuve de l'argent, Paris, Hachette; Pascale Moulévrier, 2002, Le Mutualisme bancaire. Le Crédit Mutuel de l'église au marché, Rennes, PUR; David Courpasson, 1994, «Marché concret et identité professionnelle locale. La construction de l'identité par le rapport au marché», Revue française de sociologie, 35-5, p. 197-229; François Cusin, 2004, «La Relation bancaire en question», in Exclusion et liens financiers. L'Exclusion bancaire des particuliers, dir. Georges Gloukoviezoff, Rapport du Centre Walras, Economica; Georges Gloukoviezoff, 200I, L'Exclusion bancaire et financière des particuliers: du droit à l'accès à l'autonomie, Mémoire de DEA, Université Lumière Lyon 2, Ronéo; Gilles Lazuech et Pascale Moulévrier, 2004, Exclusion monétaire et usages sociaux de l'argent, Nantes, ronéo, 250 pages.

8 On peut se reporter aux différentes contributions suivantes: Christian Thuderoz, Vincent Mangematin et Denis Harrisson, 1999, La Confiance, approches économiques et sociologiques, Gaëtan Morin éditeur; Gilles Lazuech, 2005, La Confiance, Éditions Plein Feux, Nantes ainsi que l'article de Timothy W. Guianne (2010). 
qui est exprimé autour de la notion de confiance n'est pas de même nature que ce à quoi font référence les banquiers, même si, dans les deux cas, il y a la perception d'un risque pour celui qui s'engage dans la relation d'argent. La confiance entre proches se construit sur l'interconnaissance et sur des liens durables de proximité caractéristiques des formations sociales de type communautaire. En ce sens, comme nous l'aborderons dans la troisième partie, il s'agit d'une économie morale, pour dire par ces termes qu'elle est marquée par le lien plus que par le bien. Les différences entre les crédits d'établissement et les crédits d'interconnaissance apparaissent donc plus nettement même pour des prêts importants. À l'occasion des prêts entre proches chacun s'engage personnellement dans une relation dont les dimensions relationnelles et affectives sont assez bien connues par les différents protagonistes. Par exemple, il n'est pas équivalent du point de vue du prêteur, comme de l'emprunteur, de prêter 300 euros à son fils au chômage ou de le faire pour un collègue de travail qui a perdu sa carte bancaire. Les attentes vis-à-vis du débiteur ne sont pas les mêmes et l'obligation de rendre la somme prêtée non plus. Si l'on se place du côté de la banque, ou d'une institution de crédit, deux clients qui empruntent la même somme et dont le scoring est comparable sont deux clients économiquement semblables puisque la banque évalue ses clients selon une perception d’un risque encouru à partir d'une méthode standardisée et non selon la qualité de la relation que le conseiller entretient avec son client. Par ailleurs, trahir la confiance d'un proche conduit à s'exposer à un risque que l'on pourrait qualifier de moral alors que le risque est davantage économique et juridique auprès d'un établissement de crédit. Avec un proche, c'est l'ensemble de la relation qui peut être mise en jeu. Dans le cas de la relation bancaire, qui est pratiquement toujours une relation avec un étranger, la nature de la relation en rend sûrement les enjeux moins transparents, surtout si le client est éloigné socialement et économiquement de la «culture» de la banque. 


\section{UNE ÉCONOMIE «MORALE»}

Outre les différences importantes entre les crédits d'établissement et crédits d'interconnaissance nous aimerions montrer en quoi ces derniers s'insèrent dans ce que Laurence Fontaine désigne par «l'économie morale». Ce qui caractérise cette économie, par comparaison à une économie purement marchande, est qu'elle est entièrement encastrée dans l'ensemble des relations et des pratiques sociales dans lesquelles elle se déroule et qui lui donne sens. Si l'économie morale n'échappe pas au principe général de l'intérêt, cet intérêt n'est pas uniquement d'ordre économique. Par ailleurs, si au sein de l'économie de marché dans sa forme pure l'individu - transformé en une offre ou une demande - est pensé comme libre, ce n'est pas le cas de l'économie morale au sein de laquelle l'action de chacun est subordonnée à un ensemble de déterminants affectifs, relationnels, symboliques, etc. Ce sont ces déterminants que Pierre Bourdieu a pu qualifier de formes élémentaires de domination qui lient, par la dette ou le don, des personnes entre elles. Dominations affective, symbolique, parfois sociale ou politique, qui s'inscrivent dans la relation économique de proximité même si elle n'est pas directement perçue comme telle, cette position tenue par Pierre Bourdieu, remarquons-le, tranche ouvertement avec celle généralement défendue par les économistes qui attribuent les dons et les prêts entre soi à des comportements altruistes selon la logique de la maximisation des intérêts' ${ }^{9}$. Afin de montrer l'intérêt d'une approche visant à donner aux pratiques toute leur importance, nous chercherons à montrer que les prêts entre proches qui, à la différence des prêts bancaires, sont peu standardisés et peu codifiés, ne peuvent sociologiquement se comprendre que lorsqu'ils sont resitués au sein des scènes sociales dans lesquelles ils ont vu le jour (Coquery, Menant, Weber, 2006). À cette fin, nous exposerons trois cas singuliers, offrant trois éclairages qui peuvent être comparables ou comparés à d'autres scènes sociales dans lesquelles l'argent intervient.

9 Voir Gary Becker, 1981, A treatise on the family, Harvard, Harvard University Presse ainsi que les différents travaux de Luc Arrondel et André Masson, en particulier dans divers numéros de la revue Économie et Statistique sur les comportements d'épargne des ménages. 


\section{I.PRÊTER POUR CONTRÔLER}

Bernard et Murielle habitent un pavillon dans une petite commune de Vendée. Bernard était cadre administratif au Centre régional des impôts et sa femme contrôleur. À la retraite depuis 2009, le couple touche un peu plus de 3200 euros par mois. Bernard et Murielle ont eu un fils, Ludovic, 33 ans, marié et père de deux enfants. Comme d’autres, Bernard a «horreur» des dépenses inutiles: «Tu vois le gagner plus de Sarkozy, c'est bien mais pour moi la seule façon de gagner plus, c'est de dépenser moins». Pourtant le couple profite de la vie, avant notre rencontre Bernard et Murielle revenaient tout juste d'un voyage à l'île Maurice, mais rien n'est dépensé au hasard. Ici l'usage d'Internet permet de dénicher les bonnes affaires aux prix les plus imbattables: «Nous, lorsque l'on veut profiter d'une semaine de soleil et de la mer, c'est le prix qui nous guide, pas la destination ». Le renouvellement de la voiture, qui est assez fréquent, se fait selon un calcul qui vise à déterminer le meilleur moment de la revente eu égard au coût de rachat d'un véhicule neuf et des frais occasionnés par une automobile qui prend de l'âge («je les revends avant d’avoir à refaire la courroie de distribution»). Gilbert, son voisin, s'irrite parfois de devoir leur prêter des outils pour bricoler ou pour l'entretien du jardin. C'est lui qui me confiera que Bernard règle les convecteurs électriques pour que la température dans les pièces ne dépasse pas dix-sept degrés même au cœur de l'hiver. Pourtant si Bernard et Murielle comptent, soupèsent, chacune de leurs dépenses («je vais chercher mon pain à pied, pourquoi dépenser un euro d'essence pour une baguette de pain?»), ils ne sont ni avares ni pingres. Au contraire, les vertus de l'argent dépensé au plus juste les conduisent à consentir à leur fils un prêt sans intérêt :

Je ne lui donne pas l'argent, je le lui prête, c'est notifié entre nous avec un échéancier de remboursement. Je lui prête sans intérêt parce que nous on a ce qu'il nous faut et que lui ça lui fait une économie, mais de toute façon comme c'est pas un don je suis sûr qu'il va pas acheter n'importe quoi [...]. Donc ici le principe c'est d'éviter des frais inutiles [...]. Pour ma succession, c'est la même chose, j'y pense, on n'a qu'un fils, j'essaie de voir si on peut se passer de notaire, parce que ça fait encore des frais que je juge inutiles. 
Le prêt que Bernard consent à son fils relève en fait d'une double motivation: celle d'aider un fils unique pour l'acquisition de son logement en lui épargnant des frais jugés inutiles (frais de dossier, intérêts bancaires, assurances sur le prêt) mais aussi celle de lui transmettre sa façon de faire avec l’argent («Mon passe-temps, c'est de faire des économies»). En lui prêtant son argent, Bernard acquiert sur son fils un droit de contrôle, non seulement sur son usage («comme c'est pas un don, il ne va pas acheter n'importe quoi») mais aussi sur le budget du couple puisque l'échéancier de remboursement a été fixé en commun après l'évaluation du revenu du ménage et de ses dépenses ${ }^{10}$. En amenant son fils à rembourser sur dix ans ce qu'il aurait normalement emprunté sur vingt ans auprès d'une banque, Bernard et sa femme contraignent le jeune couple à faire la chasse aux dépenses inutiles. Pour Ludovic, c'est le prix à payer pour avoir la confiance de ses parents et profiter d'un prêt sans intérêt.

\subsection{PRÊTER POUR NE PAS DONNER}

La transformation de prêt en don dans l'espace du quotidien est assez fréquente, particulièrement entre parents et enfants. Parfois cette transformation est déjà incluse, du moins implicitement, au moment où s'effectue le prêt. Ainsi Léone, 70 ans, ancienne visiteuse médicale, qui habite un petit village dans un département du Nord de la France accorde à sa fille unique, mère de trois enfants et dont le mari est au chômage depuis 24 mois, un prêt de I5 000 euros en 20I I afin que cette dernière puisse s'acheter une voiture neuve.

Je ne pouvait pas laisser Nathalie avec sa vieille voiture, elle doit faire $30 \mathrm{~km}$ tous les jours pour amener ses enfants à l'école. Cet hiver il y a eu beaucoup de neige, la voiture était souvent en panne. J’avais peur aussi pour mes petits enfants, qu'il y ait un accident, alors je me suis dis «je vais leur prêter de quoi s'acheter une voiture neuve » [...] Virgile (son gendre) m’a dit «Léone, dès que l'on pourra on te remboursera», je lui ai répondu «gardez votre argent pour l'instant, vous me remboursez plus tard, si j’arrive pas à payer la maison de retraite»

I0 C'est cette dépendance qu'évoque William Shakespeare dans Le Marchand de Venise, où le débiteur qui emprunte à ses proches est aussi redevable d'une dette morale. 
Pour comprendre le sens de cette transaction qui n'est ni un prêt (il n'y a pas d'intérêt, pas d'échéancier, pas d'obligation de rendre), ni un don (la probabilité de rendre en cas de besoin n'est pas exclue même si elle est différée dans le temps), il faut en passer par la «scène sociale» qui lui donne sens. Léone ne donne pas de l'argent à sa fille pour qu'elle achète sa voiture, elle propose de l'argent pour que le couple achète un véhicule sûr dans un souci de protection de ses petits-enfants. Ce que sa fille aurait pu accepter comme un don (parce qu'elle est fille unique), son gendre, au chômage, ne peut l'admettre et préfère que la transaction prenne, du moins symboliquement, la forme d'un prêt qui porte l'obligation de rendre même si, en réalité, la venue de cette possibilité est très peu probable.

Dans le cours des échanges domestiques, beaucoup de prêts se transforment en don, en totalité ou partiellement, au bout d'un certain temps. Certaines formules comme «tu me rembourseras quand tu pourras » ou «y'a pas d'urgence, de toute façon je n’ai pas besoin de cet argent-là » ou encore «il est plus utile à toi qu'à moi », témoignent souvent d'un don qui ne dit pas son nom même s'il n'est que partiel. Dans ces situations, l'une des questions qui reste posée est de savoir pourquoi cette transformation a lieu et pourquoi les prêts qui deviennent des dons n’avaient pas ce caractère dès le départ? Le «prêt-don » consentit par Léone à sa fille peut nous aider à comprendre ce type d'échange que l'on ne rencontre que dans les relations entre proches: Léone ne tient pas à diminuer un couple déjà fragilisé économiquement en donnant l'impression de se porter à son secours. Donner l'argent aurait contribué à diminuer symboliquement un gendre qui souffre de ne plus travailler alors même qu'il assurait jusque là un revenu très confortable à sa famille, mais prêter cet argent n’aurait pas eu beaucoup de sens puisque Léone l'avait déjà destiné à sa fille en le plaçant sur un compte d’assurance-vie dont-elle était la seule bénéficiaire. Le «prêt-don» doit donc être considéré comme l'expression de formes d'arrangement que trouvent de nombreuses familles dans des situations parfois un peu complexes. Par exemple, lorsqu'il s'agit d'aider un membre de la fratrie sans donner l'impression de désavantager les autres membres, certains parents, voire grands-parents, ont recours au «prêt-don». Ils prêtent sachant qu'ils ne seront jamais remboursés (parfois ils ne le souhaitent pas) 
mais ils conservent une trace de ce qui a été donné pour le jour ou «il faudra régler les comptes » (en cas de décès par exemple) :

On a prêté 50000 euros à notre fils qui a maintenant 35 ans pour l'aider à s'acheter un appartement, on ne lui demande rien, on est à la retraite et l'argent, si on en a, c'est pour nos jeunes [...]. Ingrid (sa fille) est au courant, elle n'a pas voulu que son frère nous signe une reconnaissance de dette, mais on a noté sur un cahier ce qu'on lui a donné [...]. C'est important pour nous de faire ça, on ne veut pas avantager l'un plus que l'autre, donc on note. (Retraitée, 63 ans, ancienne employée à la Poste).

Pour aller au-delà de la diversité des explications possibles, chacune étant liée au contexte dans lequel les transactions s'effectuent, cette catégorie d'échanges semble obéir à une sorte de dénominateur commun: elle lie sans contraindre. Alors que le don «pur» lie de façon symbolique ceux qui sont en relation et que le prêt les lie de façon économique - dans les deux types d'échanges la contrainte est au cœur de la transaction et l'obligation de rendre est forte -le «prêt-don» met en tension ces deux obligations ce qui a pour effet de les réduire sinon les annuler. Ainsi, dans le dernier exemple, ce n’est pas le fils qui contracte une dette envers ses parents, ce sont les parents qui se mettent dans l'obligation de donner, un jour ou l'autre, une somme équivalente à leur fille. Cette forme particulière d'arrangement qu'est le «prêt-don » semble constituer une solution couramment adoptée pour toutes sortes de situations concrètes au sein des familles. Elle permet de mieux comprendre en quoi la réalité est plus complexe que certaines analyses, en particulier celles généralement proposées par les économistes sur ce sujet, lorsqu'ils évoquent la question des comportements altruistes des agents sans se poser la question des conditions sociales qui permettent à ces comportements de se manifester.

\subsection{DONNER POUR RESTER LIER}

Les situations de divorce ou de rupture des couples ayant encore des enfants à charge sont de plus en plus fréquentes. Le juge aux affaires familiales dans les situations les plus courantes fixe le montant d'une pension alimentaire à verser au parent gardien (dans $77 \%$ des cas la résidence des enfants mineurs est fixée chez la mère). Si la plupart des pères acceptent le principe d'une contribution 
aux frais d'entretien et d'éducation des enfants, certains essaient de minorer le montant de la pension alimentaire en ne déclarant pas la totalité de leurs revenus au juge, voire en refusant de payer la pension (Gollac, Neuberg, Steinmetz, 20 I I). La question du montant et/ou du règlement des pensions alimentaires représente d'ailleurs prés de $64 \%$ des conflits que les couples divorcés portent en Cour d’appel (Raffin, 20I I). Pourtant, même si les situations évoquées sont assez peu fréquentes, les pères n’ayant que le «droit de visite et d’hébergement» et qui contestent la pension alimentaire font, assez fréquemment, des dons ou des prêts d'argent à leurs enfants, parfois même en les dissimulant à leur nouvelle compagne. Philippe, 57 ans, illustre cette situation. Artisan plombier exerçant son métier en Loire-Atlantique, il est le père d’Alizée, jeune femme de 22 ans, inscrite en Master 2 d'archéologie à l'université de Montpellier-III :

Avec Martine [son ex-femme], c'était la guerre permanente. Au moment du divorce, elle a voulu me faire payer notre séparation en demandant au juge une somme extravagante parce qu'elle avait choisi de revenir dans le Sud, région dont elle est originaire. Notre fille avait 8 ans, non seulement je ne la voyais plus mais en plus je payais une pension qui en fait servait surtout à entretenir sa mère $[\ldots]$ Alors, j’ai changé de stratégie, j’ai accepté beaucoup plus de chantiers au «noir», ce qui ma permit de diminuer significativement mes revenus, j'ai fait alors un recours au JAF et la pension a été fortement revue à la baisse $[. .$.$] Après, quand je voyais ma fille, à l'occasion de vacances scolaires,$ on faisait ensemble le tour des magasins, je lui payais aussi ses leçons d'équitation, un stage aussi [... ]. Lorsqu'elle a été, en 2005 puis 2006, sélectionnée au championnat de France d'équitation à Lamotte Beuvron, c'est elle qui a voulu que je l'accompagne et ça été une semaine très forte [...]. Pour l'achat de sa voiture, c'est pareil, elle m’a demandé de l'aider [...] alors je suis venu trois jours à Montpellier et on l'a cherchée ensemble [...] Progressivement, je ne dirais pas que l'argent est devenu un lien entre nous, mais ça a été important parce qu'Alizée savait que, sur ce point là, elle pouvait compter sur moi, beaucoup plus que sur sa mère qui a toujours été un panier percé, et puis aussi parce que c'est un sujet avec lequel on pouvait parler ensemble sans gène.

Dans le cas de Philippe, les prêts et dons d'argent consentis à sa fille font vivre une paternité pratique au sein de laquelle l'argent constitue un lien, un mode de partage, qu'il n'a pas pu avoir plus significativement compte tenu de la distance géographique qui les sépare. Beaucoup de pères construisent ou 
reconstruisent un lien avec leurs enfants (mineurs ou majeurs) en s'appuyant sur cette forme de transaction que sont les prêts et les dons d'argent sans que l'on ne puisse affirmer que la relation soit «intéressée » ou, plus précisément, que la principale motivation de la relation (du côté des enfants en particulier) soit les dons d’argent (Cadolle, 2005; Martial, 2005). La séparation avec les enfants, que favorise l'éloignement d'un des deux parents de leur quotidienneté, construit des configurations familiales particulières qui sont importantes à prendre en compte pour comprendre le sens sociologique que renferment ces pratiques d'argent. Lorsque Jacques, autre père divorcé, hérite d'une maison de famille, il demande à ses trois enfants s'ils sont d'accords pour devenir avec lui propriétaires. Dans ce cas, le don fait par Jacques à ses enfants, en cédant son droit de propriété exclusif sur ce bien, ne s'explique que parce qu’il désire garder - et vivre - quelque chose en commun avec ses enfants.

\section{CONCLUSION}

Il existe des formes assez variées de transactions monétaires qui semblent spécifiques à l'économie domestique. Ces relations monétaires entre proches se caractérisent par des règles ou des conventions hors marché qui sont négociées ou établies au sein des familles ou avec des amis, parfois de façon implicite surtout pour les petites sommes. Les différentes pratiques de prêts entre soi contiennent sûrement, plus que ceux consentis par les banques et les institutions de crédit, une dimension morale qui est à relier à la question du «vivre ensemble» et à celle de la parenté ou de l'amitié en pratique comme nous avons eu l'occasion de le souligner.

Face à la diversité des registres de justification des prêts entre proches, dont les trois cas présentés n’offrent qu'un aperçu (et qui, par ailleurs, sont des prêts importants), la question qui serait de savoir comment caractériser ces échanges se pose certainement. Ces relations sont-elles plutôt de type marchand? En particulier si le débiteur doit rembourser ce qu'il a emprunté, s'il est tenu de respecter un échéancier et s'il doit s'acquitter d'un taux d'intérêt. Ces relations sont-elles des dons déguisés? En particulier lorsque l'obligation de rendre est peu contraignante voire vaguement évoquée. Nous partirons de l'idée qu'il ne 
peut y avoir de réponse a priori à ces questions sans que ne soit procédé à un examen préalable des conditions sociales de ces échanges et à leur évolution dans le temps. Si l'on s'appuie sur le premier cas présenté (celui de Bernard et Murielle), la transaction semble plutôt de type marchand et, dans le second exemple (celui de Léone) elle relèverait de la catégorie des dons. Pourtant aucune de ces deux transactions n'est purement marchande ou purement un don. Dans le premier cas le lien d'interconnaissance qui relie l'emprunteur au créancier (Bernard à son fils) explique l'échange et ce lien durera après le remboursement du prêt. Dans le second cas il n'y a pas don tant que le prêteur ne renoncera pas définitivement à la possibilité de réclamer à l'emprunteur la somme prêtée. En réalité, dans la quasi-totalité des situations rencontrées lors de nos enquêtes de terrain, ni l'emprunteur ni le prêteur ne souhaitaient que ces prêts s'apparentent trop à des transactions marchandes ou à des dons. Ceci à chaque fois que la relation engageait plus de personnes que les deux protagonistes à l'échange. La qualification et, plus généralement, la compréhension de cette catégorie de prêts invitent le chercheur à envisager l'ensemble de ceux qui sont dans des relations d'interconnaissance. Par exemple si un membre de la parentèle bénéficie de certains avantages lorsqu'il emprunte dans la famille - souplesse dans les modalités de remboursement, moindre coût du crédit, etc. - des inconvénients existent aussi. Il y a parfois le «regard» des autres sur son budget et ses dépenses, il y a aussi le risque de contracter une dette morale envers le prêteur et, par extension, envers certains membres de la famille. Le don pur est souvent évité du moins dans les propos (ou alors il entre dans la logique de la transmission du patrimoine). En particulier lorsque des parents ont plusieurs enfants et que ces derniers attendent eux aussi leur part et/ou leur tour. Dès lors, il nous semble que la plupart des familles s'organisent afin que des transactions d'argent entre ses membres puissent se faire sans que cela ne nuise au «vivre ensemble». Le temps est ici une variable importante à considérer. Lorsqu'un prêt se transforme en don, c'est souvent que les conditions sociales et économiques de la famille le permettent. On peut évoquer le cas de cette mère de 74 ans, veuve depuis 2 ans, qui depuis des années prêtait régulièrement de l'argent à l'un de ses fils, architecte mais sans emploi. Cette femme a 
pu transformer les diverses créances de son fils en don à l'occasion de l'arrivée à échéance d'un produit d'assurance vie qu'elle destinait à ses trois enfants.

Les prêts et dons d'argent entre proches constituent donc des actes courants qui s'inscrivent dans la quotidienneté des familles et des relations amicales. L'importance et la fréquence de ces prêts ont été assez peu étudiées au sein même des familles du moins selon une démarche de type ethnographique. La plupart des auteurs, sociologues comme économistes, privilégient plutôt le sens général des transferts d’argent, pour tout dire leur finalité macrosociale, et ils s'appuient presque toujours sur des données statistiques. Lorsque l'on porte un regard plus «intimiste» sur les relations d'argent dans le cadre domestique, on observe qu'elles se tissent aussi avec des histoires de famille, qu'elles disent beaucoup des relations intimes qui existent ou préexistent en leur sein. C'est pourquoi il est possible d'avancer que leur relative invisibilité n'est peut-être pas uniquement liée à une difficulté singulière qu'aurait le chercheur à les objectiver et/ou au fait que ces transactions ne seraient pas soumises à des obligations légales de déclaration auprès de l’administration fiscale.

\section{BIBLIOGRAPHIE}

BIGOT Régis, 2007, Le Baromètre des solidarités familiales en France, CREDOC, collection des rapports, 246, juillet.

BLANC Jérôme, 2008, «Usages de l'argent et pratiques monétaires», working paper 2008-3, Université Lumière Lyon 2, mai.

BOLTANSKI Luc, THÉVENOT Laurent, 199I, De la justification. Les économies de la grandeur, Paris, Gallimard.

BOURDIEU Pierre, 1976, «Les modes de domination», Actes de la recherche en sciences sociales, 2, p. 122-132.

CADOLLE Sylvie, 2005, «C'est quand même mon père», Terrain, 45, Septembre.

CHANTELAT Pascal, 2002, «La nouvelle Sociologie Economique et le lien marchand: des relations personnelles à l'imperonnalité des relations», Revue Française de Sociologie, 43-3, p.521-556. 
COQUERY Natacha, MENANT François, WEBER Florence (dir.), 2006, Écrire, compter, mesurer. Vers une histoire des rationalités pratiques, Paris, Rue d'Ulm.

COURPASSON David, 1995, La Modernisation bancaire. Sociologie des rapports professions-marché, Paris, L'Harmattan.

DURKHEIM Émile, 2005, Les Règles de la méthode sociologique, Paris, Payot.

FONTAINE Laurence, 2008, L'Économie morale, Paris, Gallimard.

FOUGEYROLLAS-SCHWEBE Dominique, 1994, «L'entraide familiale: de l'universel au particulier», Sociétés Contemporaines, 17, mars, p. 51-73.

GLÉMAIN Christian (dir.), 2010, Analyse interdisciplinaire des expérimentations locales du microcrédit local, Rapport d'étude remis au Haut Commissariat aux solidarités actives, ronéo, janvier.

GOLLAC Sybille, NEUBERG Samuel, STEINMETZ Hélène, 20II, «Comment contribuer à l'entretien des enfants? Résistances à la norme judiciaire de la pension alimentaire, Communication au colloque Les Usages Sociaux de l'Argent, Nantes, 6-7 juin.

GUIANNE Timothy, 2010, «Les économistes, le crédit et la confiance», Genèses, 79, juin.

LAZUECH Gilles, 2012, L'Argent du quotidien. Les usages ordinaires de l'argent, Rennes, Les PUR.

MARTIAL Agnès, 2005, «Comment rester liés?», Terrain, 45, p. 67-82.

PASSERON Jean-Claude, REVEL Jacques (dir.), 2005, Penser par cas, Paris, EHESS.

POLANYI Karl, 1994, La Grande Transformation, Paris, Gallimard.

RAFIN Nicolas, 20II, «Les fondements de la régulation judiciaire de l'argent du divorce: du maintien social des divorçants à la moralisation des usages de l'argent», Communication au colloque Les Usages Sociaux de l'Argent, Nantes, 6-7 juin.

RIVAUD-DANSET Dominique, 1996, «Les Contrats de crédit dans une relation de long terme. De la main invisible à la poignée de main», Revue économique, 47-4, p. 937-962. 
TESTART Alain, 200I, «Échange marchand, échange non marchand», Revue Française de Sociologie, 42.

WEBER Florence, 2005, Le Sang, le Nom, le Quotidien. Une sociologie de la parenté pratique, Paris, Aux lieux d'être.

-, 2006, «Séparation des scènes sociales et pratiques ordinaires du calcul. À la recherche des raisonnements indigènes », in Natacha Coquery, François Menant, Florence Weber, op.cit, p. 66-85.

- et DUFY Caroline, 2007, L'Ethnographie économique, Paris, La Découverte.

ZELIZER Viviana, 2005, «Intimité en économie», Terrain, 45, Septembre. 